\title{
Deuterated Enzalutamide
}

National Cancer Institute

\section{Source}

National Cancer Institute. Deuterated Enzalutamide. NCI Thesaurus. Code C158096.

A deuterated form of enzalutamide, an orally bioavailable, organic, non-steroidal small molecule targeting the androgen receptor (AR) with potential antineoplastic activity. Upon administration, deuterated enzalutamide competitively binds to and inhibits the activity of ARs expressed on prostate cancer cells, which impairs nuclear translocation and DNA binding, resulting in apoptosis of prostate cancer cells. This results in a reduction in prostate cancer cell growth. AR overexpression in prostate cancer represents a key mechanism associated with prostate cancer hormone resistance. Deuterium incorporation, by replacing the hydrogen atoms of the $\mathrm{N}-\mathrm{CH} 3$ moiety with deuterium atoms, decreases enzalutamide's metabolism and allows for an increased pharmacokinetic profile, thereby enhancing its anti-tumor efficacy compared to nondeuterated enzalutamide. As the deuterated form can't cross the blood-brain barrier (BBB), the deuterated form also reduces the unwanted brain-related side effects of enzalutamide and improves its safety profile. 